PROCEEDINGS OF THE

AMERICAN MATHEMATICAL SOCIETY

Volume 139, Number 4, April 2011, Pages 1397-140

S 0002-9939(2010)10604-4

Article electronically published on September 15, 2010

\title{
GEOMETRIC VERSIONS OF SCHWARZ'S LEMMA FOR QUASIREGULAR MAPPINGS
}

\author{
DIMITRIOS BETSAKOS
}

(Communicated by Mario Bonk)

\begin{abstract}
We prove monotonicity and distortion theorems for quasiregular mappings defined on the unit ball $\mathbb{B}^{n}$ of $\mathbb{R}^{n}$. Let $K_{I}(f)$ be the inner dilatation of $f$ and let $\alpha=K_{I}(f)^{1 /(1-n)}$. Let $m_{n}$ denote $n$-dimensional Lebesgue measure and $c_{n}$ be the reduced conformal modulus in $\mathbb{R}^{n}$. We prove that the functions $r^{-n \alpha} m_{n}\left(f\left(r \mathbb{B}^{n}\right)\right)$ and $r^{-\alpha} c_{n}\left(f\left(r \mathbb{B}^{n}\right)\right)$ are increasing for $0<r<1$. These results can be viewed as variants of the classical Schwarz lemma and as generalizations of recent results by Burckel et al. for holomorphic functions in the unit disk.
\end{abstract}

\section{INTRODUCTION}

Let $\mathbb{D}=\{z \in \mathbb{C}:|z|<1\}$ be the unit disk in the complex plane $\mathbb{C}$ and for $r>0$, let $r \mathbb{D}:=\{z \in \mathbb{C}:|z|<r\}$. Let $f$ be a function holomorphic in $\mathbb{D}$. Landau and Toeplitz $([8,[5])$ proved a variant of the classical Schwarz's lemma: If $\operatorname{Diam}(f(\mathbb{D}))=2$, then for $0<r<1, \operatorname{Diam}(f(r \mathbb{D})) \leq 2 r$. Here and below, $\operatorname{Diam}(A)$ is the diameter of the set $A$. In a recent article [5], Burckel, Marshall, Minda, PoggiCorradini, and Ransford introduced a new point of view for Schwarz's lemma, the Landau-Toeplitz theorem, and related results. They proved that the function

$$
\Phi_{D}(r)=\frac{\operatorname{Diam}(f(r \mathbb{D}))}{r}, \quad 0<r<1,
$$

is increasing. This easily implies the Landau-Toeplitz theorem. They also proved some similar monotonicity results involving the area and the logarithmic capacity $C_{2}$ of the image of disks under $f$ : Each of the functions

$$
\Phi_{A}(r)=\frac{\operatorname{Area}(f(r \mathbb{D}))}{\pi r^{2}}, \quad \Phi_{C}(r)=\frac{C_{2}(f(r \mathbb{D}))}{r}, \quad 0<r<1,
$$

is increasing. Moreover, these functions are strictly increasing except when $f$ is linear (that is, of the form $f(z)=a z+b$ with $a, b \in \mathbb{C}$ ), in which case they are all constant functions.

The proofs in [5] use complex analytic arguments, as well as geometric inequalities and tools from real analysis. In the present paper, we propose a new approach. We will use conformal invariants (the conformal capacity of condensers, the Green function, the modulus metric $\mu_{G}$ ) and their behavior under suitable types

Received by the editors February 16, 2010 and, in revised form, April 26, 2010.

2010 Mathematics Subject Classification. Primary 30C65, 30C80.

Key words and phrases. Quasiregular mapping, Schwarz's lemma, capacity, conformal modulus, extremal length, symmetrization, diameter.

(C)2010 American Mathematical Society Reverts to public domain 28 years from publication 
of symmetrization (Schwarz and Steiner). This approach leads to generalizations for quasiregular mappings in higher dimensions. As expected, the results in the quasiregular case are not as sharp as for holomorphic functions.

Let $\mathbb{R}^{n}, n \geq 2$, be the $n$-dimensional Euclidean space. For $r>0$, set $r \mathbb{B}^{n}:=$ $\left\{x \in \mathbb{R}^{n}:|x|<r\right\}$ and $1 \mathbb{B}^{n}=\mathbb{B}^{n}$. We denote by $m_{n}$ the $n$-dimensional Lebesgue measure. Let $\Omega_{n}=m_{n}\left(\mathbb{B}^{n}\right)$; denote also by $\omega_{n-1}$ the $(n-1)$-dimensional surface area of the unit sphere $S^{n-1}$ in $\mathbb{R}^{n}$. Quasiregular mappings are generalizations of holomorphic functions and they are defined on domains in $\mathbb{R}^{n}$. We will use only the most basic (though highly non-trivial) properties of quasiregular mappings. We denote by $K_{I}(f)$ the inner dilatation of a quasiregular mapping $f$. For the various definitions and the properties of quasiregular mappings, we refer to [10, [14], [17. In the sequel, we assume tacitly that every quasiregular mapping that we consider is non-constant.

Theorem 1 (Volume version of Schwarz's lemma). Suppose that $f: \mathbb{B}^{n} \rightarrow \mathbb{R}^{n}$ is a quasiregular mapping and let $\alpha=K_{I}(f)^{1 /(1-n)}$.

(a) The function

$$
\Phi_{V}(r)=\frac{m_{n}\left(f\left(r \mathbb{B}^{n}\right)\right)}{r^{n \alpha}}, \quad 0<r<1,
$$

is increasing.

(b) If $m_{n}\left(f\left(\mathbb{B}^{n}\right)\right)=\Omega_{n}$, then $m_{n}\left(f\left(r \mathbb{B}^{n}\right)\right) \leq \Omega_{n} r^{n \alpha}, \quad 0<r<1$.

Note that for a holomorphic function on $\mathbb{D}$, Theorem 1 reduces to the area version of Schwarz's lemma proven in [5. We will prove Theorem 11 in section 2 and we will see that the function $\Phi_{V}$ is constant when $f$ is a radial mapping of the form $f(x)=|x|^{b-1} x, 0<b<1$. In section 2, we will also give a new proof of the strict monotonicity in the area version of Schwarz's lemma.

In section 3 we will prove a capacity version of Schwarz's lemma for quasiregular mappings. For a compact set $L$ in $\mathbb{R}^{n}$, we denote by $c_{n}(L)$ the reduced conformal modulus of $L$. This quantity, defined in detail in section 3, will play the role of logarithmic capacity in higher dimensions. For $n=2$, it is equal to the logarithmic capacity of $L$; also, in all dimensions, $c_{n}\left(r \mathbb{B}^{n}\right)=r$. If $G$ is an open bounded set in $\mathbb{R}^{n}$, then $c_{n}(G)$ will be the reduced conformal modulus of the closure $\bar{G}$ of $G$.

Theorem 2 (Capacity version of Schwarz's lemma). Suppose that $f: \mathbb{B}^{n} \rightarrow \mathbb{R}^{n}$ is a quasiregular mapping and let $\alpha=K_{I}(f)^{1 /(1-n)}$.

(a) The function

is increasing.

$$
\Phi_{C}(r)=\frac{c_{n}\left(f\left(r \mathbb{B}^{n}\right)\right)}{r^{\alpha}}, \quad 0<r<1,
$$

(b) If $c_{n}\left(f\left(\mathbb{B}^{n}\right)\right)=1$, then $c_{n}\left(f\left(r \mathbb{B}^{n}\right)\right) \leq r^{\alpha}, \quad 0<r<1$.

If $f$ is a holomorphic function on $\mathbb{D}$, Theorem 2 reduces to the logarithmic capacity version of Schwarz's lemma, originally proven in [5. In section 3] we will also give a new proof of strict monotonicity in this version of Schwarz's lemma.

In section 4 we will prove a diameter version of Schwarz's lemma for quasiregular mappings. It is a distortion theorem involving the function $\gamma_{n}$. For $1<s<\infty$, $\gamma_{n}(s)$ is the conformal modulus of the family of curves joining the complementary components $\overline{\mathbb{B}}^{n}$ and $\left[s e_{1}, \infty\right]$ of the Grötzsch ring. Here $e_{1}=(1,0, \ldots, 0) \in \mathbb{R}^{n}$. The function $\gamma_{n}$ plays an important role in the distortion theory of quasiconformal and quasiregular mappings; see [2, [17. 
Theorem 3 (Diameter version of Schwarz's lemma). Suppose that $f: \mathbb{B}^{n} \rightarrow \mathbb{R}^{n}$ is a quasiregular mapping. For $0<r<s<1$,

$$
\gamma_{n}\left(\frac{\tilde{r}^{2}+\tilde{s}^{2}}{2 \tilde{r} \tilde{s}}\right) \leq K_{I}(f) \gamma_{n}\left(\frac{r^{2}+s^{2}}{2 r s}\right),
$$

where $2 \tilde{r}=\operatorname{Diam}\left(f\left(r \mathbb{B}^{n}\right)\right)$ and $2 \tilde{s}=\operatorname{Diam}\left(f\left(s \mathbb{B}^{n}\right)\right)$. If $\operatorname{Diam}\left(f\left(\mathbb{B}^{n}\right)\right)=2$, then

$$
\gamma_{n}\left(\frac{\tilde{r}^{2}+1}{2 \tilde{r}}\right) \leq K_{I}(f) \gamma_{n}\left(\frac{r^{2}+1}{2 r}\right) .
$$

If $f$ is holomorphic on $\mathbb{D}$, then setting $n=2$ and $K_{I}(f)=1$ and using properties of the function $\gamma_{2}$, we obtain the inequality of the Landau-Toeplitz theorem and the corresponding monotonicity result of [5]. We give a new proof of the strict monotonicity result in section 4 .

Monotonicity results in the theory of quasiregular mappings, related to Theorems 113, appear in [13, p. 82] and [1, p. 698].

\section{The volume Version of Schwarz's Lemma}

2.1. Condensers. Schwarz symmetrization. For the proof of Theorem 1 we will use Schwarz symmetrization and its action on the conformal capacity of condensers. We refer to [9, [10, [15, [17, for the facts about condensers that we will need in the proof. A condenser is a pair $(A, C)$, where $A$ is an open set in $\mathbb{R}^{n}$ and $C$ is a compact subset of $A$. We will denote by $\operatorname{cap}(A, C)$ the conformal capacity of the condenser $(A, C)$. A spherical ring is a condenser of the form $\left(s \mathbb{B}^{n}, \overline{r \mathbb{B}^{n}}\right)$ with $0<r<s$. The conformal capacity of a spherical ring can be computed explicitly:

$$
\operatorname{cap}\left(s \mathbb{B}^{n}, \overline{r \mathbb{B}^{n}}\right)=\omega_{n-1}\left(\log \frac{s}{r}\right)^{1-n} .
$$

The Schwarz symmetrization of an open set $A$ with $m_{n}(A)<\infty$ is the ball $A^{\sharp}$ centered at the origin with $m_{n}\left(A^{\sharp}\right)=m_{n}(A)$. The Schwarz symmetrization of a compact set $C$ is similarly the closed ball $C^{\sharp}$ centered at the origin with $m_{n}\left(C^{\sharp}\right)=m_{n}(C)$. The Schwarz symmetrization of the condenser $(A, C)$ is the spherical ring $\left(A^{\sharp}, C^{\sharp}\right)$. It is known (see [15]) that Schwarz symmetrization decreases the capacity of condensers:

$$
\operatorname{cap}\left(A^{\sharp}, C^{\sharp}\right) \leq \operatorname{cap}(A, C) .
$$

Proof of Theorem 1. Let $0<r<s<1$. Let $f: \mathbb{B}^{n} \rightarrow \mathbb{R}^{n}$ be quasiregular. The map $f$ is an open mapping, and hence the pair $\left(f\left(s \mathbb{B}^{n}\right), \overline{\left.f\left(r \mathbb{B}^{n}\right)\right)}\right.$ is a condenser. Moreover, by a fundamental theorem in the theory of quasiregular mappings [10, Theorem 7.1],

$$
\operatorname{cap}\left(f\left(s \mathbb{B}^{n}\right), \overline{f\left(r \mathbb{B}^{n}\right)}\right) \leq K_{I}(f) \operatorname{cap}\left(s \mathbb{B}^{n}, \overline{r \mathbb{B}^{n}}\right) .
$$

Let $f\left(s \mathbb{B}^{n}\right)^{\sharp}=s^{\sharp} \mathbb{B}^{n}$ and $\overline{f\left(r \mathbb{B}^{n}\right)^{\sharp}}=\overline{r^{\sharp} \mathbb{B}^{n}}$.

By (2.1), (2.2), and (2.3),

$$
\begin{aligned}
\omega_{n-1}\left(\log \frac{s^{\sharp}}{r^{\sharp}}\right)^{1-n} & \left.=\operatorname{cap}\left(s^{\sharp} \mathbb{B}^{n}, \overline{r^{\sharp} \mathbb{B}^{n}}\right) \leq \operatorname{cap}\left(f\left(s \mathbb{B}^{n}\right), \overline{f\left(r \mathbb{B}^{n}\right.}\right)\right) \\
& \leq K_{I}(f) \operatorname{cap}\left(s \mathbb{B}^{n}, \overline{r \mathbb{B}^{n}}\right)=K_{I}(f) \omega_{n-1}\left(\log \frac{s}{r}\right)^{1-n} .
\end{aligned}
$$


Hence

$$
\log \frac{s}{r} \leq K_{I}(f)^{1 /(n-1)} \log \frac{s^{\sharp}}{r^{\sharp}},
$$

which is equivalent to the inequality $\Phi_{V}(r) \leq \Phi_{V}(s)$. So part (a) of the theorem is proved. Part (b) follows easily from part (a).

2.2. The radial mapping. Let $0<b<1$ and consider the function $f: \mathbb{B}^{n} \rightarrow \mathbb{R}^{n}$ with

$$
f(x)= \begin{cases}|x|^{b-1} x, & x \in \mathbb{B}^{n} \backslash\{0\}, \\ 0, & x=0 .\end{cases}
$$

Then [16, §16.2] $f$ is a quasiconformal mapping with $K_{I}(f)=b^{1-n}$. Therefore $\alpha:=K_{I}(f)^{1 /(1-n)}=b$. Moreover, if $0<\rho<1$, then $f\left(\rho \mathbb{B}^{n}\right)=\rho^{b} \mathbb{B}^{n}$. Hence for $0<r<s<1$,

$$
\frac{m_{n}\left(f\left(s \mathbb{B}^{n}\right)\right)}{m_{n}\left(f\left(r \mathbb{B}^{n}\right)\right)}=\frac{m_{n}\left(s^{b} \mathbb{B}^{n}\right)}{m_{n}\left(r^{b} \mathbb{B}^{n}\right)}=\frac{s^{n b}}{r^{n b}} .
$$

So for this function $f$, we have that $\Phi_{V}$ is constant.

2.3. The area version of the Schwarz lemma. We give a new proof of the following theorem.

Theorem $4([5])$. Suppose that $f$ is holomorphic on $\mathbb{D}$. The function $\Phi_{A}(r):=$ $\left(\pi r^{2}\right)^{-1} m_{2}(f(r \mathbb{D}))$ is strictly increasing for $0<r<1$, except when $f$ is linear, in which case $\Phi_{A}$ is constant.

Proof. We may assume that $f(0)=0$. Setting $n=2$ and $K_{I}(f)=1$ in Theorem 1. we see that $\Phi_{A}$ is increasing. Suppose that $\phi_{A}(r)=\phi_{A}(s)$ for some $r, s$ with $0<r<s<1$. Then it follows from the proof of Theorem 1 that $r^{\sharp} / r=s^{\sharp} / s$ and that

$$
\operatorname{cap}\left(s^{\sharp} \mathbb{D}, \overline{r^{\sharp} \mathbb{D}}\right)=\operatorname{cap}(f(s \mathbb{D}), \overline{f(r \mathbb{D})}),
$$

which means that we have equality in the Schwarz symmetrization inequality for the capacity of planar condensers. By a modification of the argument in [6, §3],

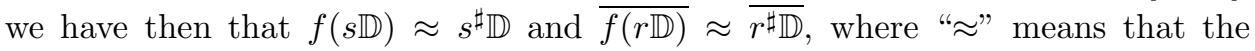
symmetric difference between the two sets has logarithmic capacity zero. The relation $f(s \mathbb{D}) \approx s^{\sharp} \mathbb{D}$ implies that $f(s \mathbb{D}) \subset s^{\sharp} \mathbb{D}$. Then by the classical Schwarz lemma, for all $z \in s \mathbb{D}$,

$$
|f(z)| \leq \frac{s^{\sharp}}{s}|z| .
$$

The relation $\overline{f(r \mathbb{D})} \approx \overline{r^{\sharp} \mathbb{D}}$ implies that for some real $\theta$,

$$
\left|f\left(r e^{i \theta}\right)\right|=r^{\sharp}=\frac{r^{\sharp}}{r} r=\frac{s^{\sharp}}{s}\left|r e^{i \theta}\right| .
$$

So we have equality in Schwarz's lemma. Therefore $f$ is linear. 


\section{The CAPACITy Version of SchWARZ'S LEMma}

3.1. The reduced conformal modulus. If $(A, C)$ is a condenser in $\mathbb{R}^{n}$, we will denote by $M_{n}(A, C)$ the conformal modulus of the family of curves in $A$ joining $C$ with $\partial A$. We will also use the extremal length of this family defined by $\lambda_{n}(A, C)=$ $M_{n}(A, C)^{1 /(1-n)}$. The conformal capacity of $(A, C)$ is defined through the Dirichlet integral and it is equal to $M_{n}(A, C)$; see [9, Appendix $\left.\mathrm{A}\right]$ and the references therein.

We define the reduced conformal modulus by a standard method. Let $L$ be a compact set in $\mathbb{R}^{n}$. Suppose that $r_{1}$ is large enough so that $L \subset r_{1} \mathbb{B}^{n}$. If $0<r_{1}<r_{2}$, then by a well-known property of extremal length (see e.g. [2, pp. 159161] or [9, Appendix A]),

$$
\begin{aligned}
\lambda_{n}\left(r_{2} \mathbb{B}^{n}, L\right) & \geq \lambda_{n}\left(r_{1} \mathbb{B}^{n}, L\right)+\lambda_{n}\left(r_{2} \mathbb{B}^{n}, \overline{r_{1} \mathbb{B}^{n}}\right) \\
& =\lambda_{n}\left(r_{1} \mathbb{B}^{n}, L\right)+\omega_{n-1}^{1 /(1-n)} \log \frac{r_{2}}{r_{1}} .
\end{aligned}
$$

Hence the function $r \mapsto \lambda_{n}\left(r \mathbb{B}^{n}, L\right)-\omega_{n-1}^{1 /(1-n)} \log r$ is increasing. Let

$$
\lambda_{n}(L):=\lim _{r \rightarrow \infty}\left(\lambda_{n}\left(r \mathbb{B}^{n}, L\right)-\omega_{n-1}^{1 /(1-n)} \log r\right) .
$$

The reduced conformal modulus of the compact set $L \subset \mathbb{R}^{n}$ is defined by

$$
c_{n}(L)=\exp \left(-\omega_{n-1}^{1 /(n-1)} \lambda_{n}(L)\right) .
$$

We will see now that for $n=2, c_{2}$ is equal to the logarithmic capacity $C_{2}$. Let $L$ be a planar compact set. If $C_{2}(L)=0$, then for every disk $r \mathbb{D}$ containing $L$, we have $\operatorname{cap}(r \mathbb{D}, L)=0$ and therefore $c_{2}(L)=0$. Suppose that $L$ has positive logarithmic capacity. Let $g_{L}$ be the Green function of the complement of $L$. The Robin constant $\gamma(L)$ of $L$ is defined by

$$
g_{L}(z, \infty)=\log |z|+\gamma(L)+o(1), \quad z \rightarrow \infty .
$$

Then $C_{2}(L)=\exp (-\gamma(L))$. It follows from the analysis in [7, pp. 126-127] that

$$
\begin{aligned}
\frac{1}{2 \pi} \gamma(L) & =\lim _{r \rightarrow \infty}\left(\frac{1}{\operatorname{cap}(r \mathbb{D}, L)}-\frac{1}{2 \pi} \log r\right) \\
& =\lim _{r \rightarrow \infty}\left(\lambda_{2}(r \mathbb{D}, L)-\frac{1}{2 \pi} \log r\right)=\lambda_{2}(L) .
\end{aligned}
$$

Hence $C_{2}(L)=\exp \left(-2 \pi \lambda_{2}(L)\right)=c_{2}(L)$.

The reduced conformal modulus of a closed ball is equal to its radius. Indeed,

$$
\begin{aligned}
\lambda_{n}\left(\overline{\rho \mathbb{B}^{n}}\right) & =\lim _{r \rightarrow \infty}\left(\lambda_{n}\left(r \mathbb{B}^{n}, \overline{\rho \mathbb{B}^{n}}\right)-\omega_{n-1}^{1 /(1-n)} \log r\right) \\
& =\lim _{r \rightarrow \infty}\left(\omega_{n-1}^{1 /(1-n)} \log \frac{r}{\rho}-\omega_{n-1}^{1 /(1-n)} \log r\right) \\
& =-\omega_{n-1}^{1 /(1-n)} \log \rho .
\end{aligned}
$$

Hence $c_{n}\left(\overline{\rho \mathbb{B}^{n}}\right)=\exp (\log \rho)=\rho$.

3.2. A special symmetrization lemma. We will need the following lemma. We note that an analogous result with Newtonian capacity in place of the reduced conformal modulus has been proved by Zorii 18 . 
Lemma 1. Let $(G, L)$ be a condenser in $\mathbb{R}^{n}$ with $G$ bounded. Let $G^{*}$ denote the ball centered at the origin with $c_{n}\left(G^{*}\right)=c_{n}(G)$. Let $L^{*}$ denote the closed ball centered at the origin with $c_{n}\left(L^{*}\right)=c_{n}(L)$. Then

$$
\operatorname{cap}\left(G^{*}, L^{*}\right) \leq \operatorname{cap}(G, L) .
$$

Proof. Set $G^{*}=s^{*} \mathbb{B}^{n}$ and $L^{*}=\overline{r^{*} \mathbb{B}^{n}}$. Take $\rho>0$ large enough so that $G \subset \rho \mathbb{B}^{n}$. By well-known properties of extremal length (see e.g. [9] or [17]),

$$
\lambda_{n}\left(\rho \mathbb{B}^{n}, L\right) \geq \lambda_{n}\left(\rho \mathbb{B}^{n}, \bar{G}\right)+\lambda_{n}(G, L)
$$

and

$$
\begin{aligned}
\lambda_{n}\left(\rho \mathbb{B}^{n}, L^{*}\right) & =\lambda_{n}\left(\rho \mathbb{B}^{n}, \overline{r^{*} \mathbb{B}^{n}}\right)=\omega_{n-1}^{1 /(1-n)} \log \frac{\rho}{r^{*}} \\
& =\omega_{n-1}^{1 /(1-n)} \log \frac{\rho}{s^{*}}+\omega_{n-1}^{1 /(1-n)} \log \frac{s^{*}}{r^{*}} \\
& =\lambda_{n}\left(\rho \mathbb{B}^{n}, \overline{s^{*} \mathbb{B}^{n}}\right)+\lambda_{n}\left(s^{*} \mathbb{B}^{n}, \overline{r^{*} \mathbb{B}^{n}}\right) \\
& =\lambda_{n}\left(\rho \mathbb{B}^{n}, \overline{G^{*}}\right)+\lambda_{n}\left(G^{*}, L^{*}\right) .
\end{aligned}
$$

Subtracting $\omega_{n-1}^{1 /(1-n)} \log \rho$ from both sides of (3.3) and taking limits as $\rho \rightarrow \infty$, we obtain

$$
\lambda_{n}(G, L) \leq \lambda_{n}(\bar{G})-\lambda_{n}(L)
$$

Similarly, (3.4) yields

$$
\lambda_{n}\left(G^{*}, L^{*}\right)=\lambda_{n}\left(\overline{G^{*}}\right)-\lambda_{n}\left(L^{*}\right) .
$$

The assumptions $c_{n}(G)=c_{n}\left(G^{*}\right)$ and $c_{n}(L)=c_{n}\left(L^{*}\right)$ together with (3.5) and (3.6) give

$$
\lambda_{n}(G, L) \leq \lambda_{n}\left(G^{*}, L^{*}\right),
$$

which is equivalent to (3.2).

Proof of Theorem 2 . The outer boundary of a bounded set in $\mathbb{R}^{n}$ is the boundary of the unbounded complementary component of the set. By a well-known property of extremal length (see [16, Section 11]), the reduced conformal modulus of a compact set is equal to the reduced conformal modulus of its outer boundary.

Let $0<r<s<1$. Let $L_{r}$ be the compact set bounded by the outer boundary of $f\left(r \mathbb{B}^{n}\right)$. Let $G_{s}$ be the domain bounded by the outer boundary of $f\left(s \mathbb{B}^{n}\right)$. Then

$$
c_{n}\left(f\left(r \mathbb{B}^{n}\right)\right)=c_{n}\left(L_{r}\right) \quad \text { and } \quad c_{n}\left(f\left(s \mathbb{B}^{n}\right)\right)=c_{n}\left(G_{s}\right) .
$$

Also, the pair $\left(G_{s}, L_{r}\right)$ is a condenser. Every curve joining $\partial G_{s}$ with $L_{r}$ also joins $\partial f\left(s \mathbb{B}^{n}\right)$ with $\overline{f\left(r \mathbb{B}^{n}\right)}$. Hence $M_{n}\left(G_{s}, L_{s}\right) \leq M_{n}\left(f\left(s \mathbb{B}^{n}\right), \overline{f\left(r \mathbb{B}^{n}\right)}\right)$ or, equivalently,

$$
\operatorname{cap}\left(G_{s}, L_{r}\right) \leq \operatorname{cap}\left(f\left(s \mathbb{B}^{n}\right), \overline{f\left(r \mathbb{B}^{n}\right)}\right) .
$$

Next we consider the balls $G_{s}^{*}=s^{*} \mathbb{B}^{n}$ and $L_{r}^{*}=\overline{r^{*} \mathbb{B}^{n}}$ with $c_{n}\left(G_{s}^{*}\right)=c_{n}\left(G_{s}\right)$ and $c_{n}\left(L_{r}^{*}\right)=c_{n}\left(L_{r}\right)$. By Lemma 1,

$$
\operatorname{cap}\left(G_{s}^{*}, L_{r}^{*}\right) \leq \operatorname{cap}\left(G_{s}, L_{r}\right) .
$$


By [10, Theorem 7.1], (3.9), and (3.10),

$$
\begin{aligned}
\omega_{n-1}\left(\log \frac{s}{r}\right)^{1-n} & =\operatorname{cap}\left(s \mathbb{B}^{n}, \overline{r \mathbb{B}^{n}}\right) \\
& \geq K_{I}(f)^{-1} \operatorname{cap}\left(f\left(s \mathbb{B}^{n}\right), \overline{f\left(r \mathbb{B}^{n}\right)}\right) \\
& \geq K_{I}(f)^{-1} \operatorname{cap}\left(G_{s}, L_{r}\right) \\
& \geq K_{I}(f)^{-1} \operatorname{cap}\left(G_{s}^{*}, L_{r}^{*}\right) \\
& =K_{I}(f)^{-1} \operatorname{cap}\left(s^{*} \mathbb{B}^{n}, r^{*} \mathbb{B}^{n}\right) \\
& =K_{I}(f)^{-1} \omega_{n-1}\left(\log \frac{s^{*}}{r^{*}}\right)^{1-n} .
\end{aligned}
$$

Hence

$$
\frac{s^{\alpha}}{r^{\alpha}} \leq \frac{s^{*}}{r^{*}}
$$

It follows from (3.8) and (3.12) that

$$
\begin{aligned}
\Phi_{C}(r) & =\frac{c_{n}\left(f\left(r \mathbb{B}^{n}\right)\right)}{r^{\alpha}}=\frac{c_{n}\left(L_{r}\right)}{r^{\alpha}}=\frac{c_{n}\left(L_{r}^{*}\right)}{r^{\alpha}}=\frac{r^{*}}{r^{\alpha}} \leq \frac{s^{*}}{s^{\alpha}} \\
& =\frac{c_{n}\left(G_{s}^{*}\right)}{s^{\alpha}}=\frac{c_{n}\left(f\left(s \mathbb{B}^{n}\right)\right)}{s^{\alpha}}=\Phi_{C}(s)
\end{aligned}
$$

and part (a) of the theorem is proved. Part (b) follows easily from part (a).

Remark. For the radial mapping of subsection 2.2, the function $\Phi_{C}$ is constant.

3.3. The logarithmic capacity version of Schwarz's lemma. In this subsection we restrict ourselves to dimension $n=2$ and consider a holomorphic function $f: \mathbb{D} \rightarrow \mathbb{C}$ and the function $\Phi_{C}(r):=r^{-1} C_{2}(f(r \mathbb{D}))$. It has been proved in $[5$, that $\Phi_{C}$ is strictly increasing unless $f$ is linear. It follows easily from Theorem 2 that $\phi_{C}$ is increasing. To achieve strict monotonicity, we need to modify the method of proof and use conformal mappings and properties of logarithmic capacity that can be found in [11] and [6].

Theorem 5 ([5]). Suppose that $f$ is holomorphic on $\mathbb{D}$. The function $\Phi_{C}(r):=$ $r^{-1} C_{2}(f(r \mathbb{D}))$ is strictly increasing for $0<r<1$, except when $f$ is linear, in which case $\Phi_{C}$ is constant.

Proof. Let $0<r<s<1$. Let $L_{r}$ be the compact set bounded by the outer boundary of $f(r \mathbb{D})$ and let $G_{s}$ be the simply connected domain bounded by the outer boundary of $f(s \mathbb{D})$. Then $d_{r}:=C_{2}(f(r \mathbb{D}))=C_{2}\left(L_{r}\right)$ and $d_{s}:=C_{2}(f(s \mathbb{D}))=$ $C_{2}\left(G_{s}\right)$. Moreover,

$$
\begin{aligned}
2 \pi\left(\log \frac{s}{r}\right)^{-1} & =\operatorname{cap}(s \mathbb{D}, \overline{r \mathbb{D}}) \\
& \geq \operatorname{cap}(f(s \mathbb{D}), \overline{f(r \mathbb{D})}) \geq \operatorname{cap}\left(G_{s}, L_{r}\right) .
\end{aligned}
$$

Let $D_{r}$ be the complement of $L_{r}$ in the extended complex plane $\widehat{\mathbb{C}}$ and consider the conformal mapping $g: D_{r} \rightarrow \widehat{\mathbb{C}} \backslash d_{r} \mathbb{D}$ with $g(\infty)=\infty$ and $g^{\prime}(\infty)=1$. Then $g$ maps the boundary of $G_{s}$ onto the boundary of a simply connected domain $A_{s}$ which contains the disk $d_{r} \mathbb{D}$ and $C_{2}\left(\overline{A_{s}}\right)=C_{2}\left(\overline{G_{s}}\right)=d_{s}$. By the conformal invariance of the condenser capacity,

$$
\operatorname{cap}\left(A_{s}, d_{r} \mathbb{D}\right)=\operatorname{cap}\left(G_{s}, L_{r}\right)
$$


Let $d_{s}^{\sharp} \mathbb{D}$ be the Schwarz symmetrization $A_{s}^{\sharp}$ of $A_{s}$. Then

$$
\operatorname{cap}\left(A_{s}, d_{r} \mathbb{D}\right) \geq \operatorname{cap}\left(A_{s}^{\sharp}, d_{r} \mathbb{D}\right)=2 \pi\left(\log \frac{d_{s}^{\sharp}}{d_{r}}\right)^{-1} .
$$

Schwarz symmetrization also reduces the logarithmic capacity of compact sets. Hence

$$
d_{r}^{\sharp} \leq C_{2}\left(\overline{A_{s}}\right)=d_{s} .
$$

We combine (3.13), (3.14), (3.15), and (3.16) to obtain

$$
\frac{s}{r} \leq \frac{d_{s}}{d_{r}}
$$

Therefore

$$
\Phi_{C}(r)=\frac{C_{2}(f(r \mathbb{D}))}{r}=\frac{d_{r}}{r} \leq \frac{d_{s}}{s}=\frac{C_{2}(f(s \mathbb{D}))}{s}=\Phi_{C}(s) .
$$

We have proved that $\Phi_{C}$ is increasing. Now suppose that for some $0<r<s<1$, $\Phi_{C}(r)=\Phi_{C}(s)$. Then $\Phi_{C}(\rho)=\Phi_{C}(s)$ for all $\rho$ with $r<\rho<s$. Moreover, we have equality in the Schwarz symmetrization inequality for the capacity of planar condensers. Therefore (see [6]), after a translation, $A_{s}=d_{s} \mathbb{D}$. Hence the function $g \circ f$ maps the annulus $\{r<|z|<s\}$ onto the annulus $\left\{d_{r}<|z|<d_{s}\right\}$ and each circle $\{|z|=\rho\}$ onto a circle centered at the origin. By the annulus theorem [12, Chapter 9], $g \circ f(z)=\frac{d_{s}}{s} z, \quad r<|z|<s$. Therefore

$$
f=g^{-1}\left(\frac{d_{s}}{s} z\right), \quad r<|z|<s .
$$

The function $f$ is holomorphic in $\mathbb{D}$ and the function $g^{-1}\left(d_{s} z / s\right)$ is holomorphic in $\mathbb{C} \backslash r \mathbb{D}$ with a simple pole at $\infty$. Hence the function

$$
F(z)= \begin{cases}f(z), & z \in \mathbb{D}, \\ g^{-1}\left(d_{s} z / s\right), & z \in \mathbb{C} \backslash r \mathbb{D},\end{cases}
$$

is holomorphic in $\mathbb{C}$ with a simple pole at $\infty$. It follows that $f(z)=F(z)=d_{s} z / s$ + a constant.

\section{The Diameter Version of SchwarZ's Lemma}

4.1. Steiner symmetrization. The Steiner symmetrization of an open set $G \subset$ $\mathbb{R}^{n}$ with respect to an $(n-1)$-dimensional plane $\Pi$ is the open set $G^{\boxminus}$ which is symmetric with respect to $\Pi$ and has the following property: If $\Sigma$ is a straight line perpendicular to $\Pi$, then the set $G^{\boxminus} \cap \Sigma$ is a single interval with $m_{1}\left(G^{\boxminus} \cap \Sigma\right)=$ $m_{1}(G \cap \Sigma)$. The Steiner symmetrization of a closed set is defined in an analogous way. We say that an open set $G$ is Steiner symmetric with respect to $\Pi$ if $G^{\boxminus}=G$. For detailed definitions, properties, and applications of Steiner symmetrization, we refer to [6], 7], 15, [4. It is clear that Steiner symmetrization decreases the diameter of an open or closed set. It also decreases the capacity of condensers; see [15]. We will need the following simple geometric result.

Lemma 2. Let $\Omega$ be an open set in $\mathbb{R}^{n}$ with $\operatorname{Diam}(\Omega)=d$. Suppose that $\bar{\Omega}$ contains the points $(-d / 2,0, \ldots, 0)$ and $(d / 2,0, \ldots, 0)$ and that $\Omega$ is Steiner symmetric with respect to all the $(n-1)$-dimensional planes $\left\{\left(x_{1}, x_{2}, \ldots, x_{n}\right) \in \mathbb{R}^{n}: x_{j}=0\right\}$, $j=1,2, \ldots, n$. Then $\Omega \subset \frac{d}{2} \mathbb{B}^{n}$. 
Proof. Suppose that there exists a point $x=\left(x_{1}, x_{2}, \ldots, x_{n}\right) \in \Omega$ with $|x|>d / 2$. By the symmetry assumption, the point $-x=\left(-x_{1},-x_{2}, \ldots,-x_{n}\right)$ belongs to $\Omega$. Hence

$$
\operatorname{Diam}(\Omega) \geq|x-(-x)|=2|x|>2 d / 2=d
$$

a contradiction.

4.2. The modulus metric. Let $G$ be a bounded domain in $\mathbb{R}^{n}$. For $x, y \in G$, we define

$$
\mu_{G}(x, y):=\inf _{C_{x y}} M_{n}\left(G, C_{x y}\right),
$$

where the infimum is taken over all curves $C_{x y}$ in $G$ joining $x$ and $y$. The function $\mu_{G}$ is a conformally invariant metric called the modulus metric. We list the properties of the modulus metric that we will need; for more information, see [17.

(i) If $x, y \in D \subset G$, then $\mu_{D}(x, y) \geq \mu_{G}(x, y)$.

(ii) If $f: G \rightarrow \mathbb{R}^{n}$ is quasiregular, then $\mu_{f(G)}(f(x), f(y)) \leq K_{I}(f) \mu_{G}(x, y)$; see [17, Section 10].

(iii) If $\Pi$ is an (n-1)-dimensional plane passing through $x, y \in G$, then $\mu_{G}(x, y) \geq$ $\mu_{G^{\boxminus}}(x, y)$. This comes from the definition of $\mu_{G}$ and the fact that Steiner symmetrization decreases the capacity of condensers.

(iv) If $G$ is Steiner symmetric with respect to every plane $\left\{x_{j}=0\right\}, j=$ $2,3, \ldots, n$, and both $x$ and $y$ lie on the straight line $\left\{x_{2}=x_{3}=\cdots=x_{n}=0\right\}$, then an extremal curve in the definition of $\mu_{G}$ is the linear segment joining $x$ and $y$. This follows from (iii).

(v) If $G$ is Steiner symmetric with respect to each of the planes $\left\{x_{j}=0\right\}$, $j=2,3, \ldots, n$, and $G^{\boxminus}$ is the Steiner symmetrization of $G$ with respect to the plane $\left\{x_{1}=0\right\}$, then $\mu_{G}\left(-t e_{1}, t e_{1}\right) \geq \mu_{G} \boxminus\left(-t e_{1}, t e_{1}\right)$. This comes from (iv) and the fact that Steiner symmetrization decreases the capacity of condensers.

(vi) There exist explicit formulae for $\mu_{\mathbb{B}^{n}}(x, y)$; see [17, Section 10], [2, Chapter 8]. It follows from these formulae that

$$
\mu_{\mathbb{B}^{n}}\left(-t e_{1}, t e_{1}\right)=\gamma_{n}\left(\frac{1+t^{2}}{2 t}\right) .
$$

Recall that $\gamma_{n}$ is the conformal modulus of the Grötzsch ring; see the introduction.

(vii) If $x$ and $y$ lie on the sphere $\{|x|=r\}, 0<r<1$, then $\mu_{\mathbb{B}^{n}}(x, y) \leq$ $\mu_{\mathbb{B}^{n}}\left(-r e_{1}, r e_{1}\right)$. This also follows from the formulae mentioned in (vi).

Proof of Theorem 3. Let $f: \mathbb{B}^{n} \rightarrow \mathbb{R}^{n}$ be a quasiregular mapping. Let $0<r<s<$ 1. Let $\tilde{r}:=\operatorname{Diam}\left(f\left(r \mathbb{B}^{n}\right)\right) / 2$ and $\tilde{s}:=\operatorname{Diam}\left(f\left(s \mathbb{B}^{n}\right)\right) / 2$. Let $y_{r}, \tilde{y}_{r}$ be two points on $\overline{f\left(r \mathbb{B}^{n}\right)}$ with $\operatorname{Diam}\left(\overline{f\left(r \mathbb{B}^{n}\right)}\right)=\left|y_{r}-\tilde{y}_{r}\right|$. We may assume that $y_{r}=-\tilde{r} e_{1}$ and $\tilde{y}_{r}=\tilde{r} e_{1}$. Let $x_{r}, \tilde{x}_{r}$ be points on the sphere $\{|x|=r\}$ with $f\left(x_{r}\right)=-\tilde{r} e_{1}$ and $f\left(\tilde{x}_{r}\right)=\tilde{r} e_{1}$. By the properties of the modulus metric presented in subsection 4.2.

$$
\begin{aligned}
\mu_{f\left(s \mathbb{B}^{n}\right)}\left(-\tilde{r} e_{1}, \tilde{r} e_{1}\right) & \leq K_{I}(f) \mu_{s \mathbb{B}^{n}}\left(x_{r}, \tilde{x}_{r}\right) \\
& \leq K_{I}(f) \mu_{s \mathbb{B}^{n}}\left(-r e_{1}, r e_{1}\right) \\
& =K_{I}(f) \mu_{\mathbb{B}^{n}}\left(-\frac{r}{s} e_{1}, \frac{r}{s} e_{1}\right) \\
& =K_{I}(f) \gamma_{n}\left(\frac{r^{2}+s^{2}}{2 r s}\right) .
\end{aligned}
$$


Let $\mathcal{S}_{j}$ denote Steiner symmetrization with respect to the $(n-1)$-dimensional plane $\left\{x_{j}=0\right\}$. Let

$$
\Omega_{s}:=\mathcal{S}_{1} \circ \mathcal{S}_{2} \circ \cdots \circ \mathcal{S}_{n}\left(f\left(s \mathbb{B}^{n}\right)\right) .
$$

By the properties of Steiner symmetrization and of the modulus metric presented in subsections 4.1 and 4.2 ,

$$
\begin{aligned}
\mu_{f\left(s \mathbb{B}^{n}\right)}\left(-\tilde{r} e_{1}, \tilde{r} e_{1}\right) & \geq \mu_{\Omega_{s}}\left(-\tilde{r} e_{1}, \tilde{r} e_{1}\right) \geq \mu_{\tilde{s} \mathbb{B}^{n}}\left(-\tilde{r} e_{1}, \tilde{r} e_{1}\right) \\
& =\mu_{\mathbb{B}^{n}}\left(-\frac{\tilde{r}}{\tilde{s}} e_{1}, \frac{\tilde{r}}{\tilde{s}} e_{1}\right)=\gamma_{n}\left(\frac{\tilde{r}^{2}+\tilde{s}^{2}}{2 \tilde{r} \tilde{s}}\right) .
\end{aligned}
$$

Now (1.1) follows from (4.1) and (4.2). The inequality (1.2) follows easily from (1.1) and the continuity and monotonicity of the function $\gamma_{n}$.

Remark. I do not know whether the function $r \mapsto \operatorname{Diam}\left(f\left(r \mathbb{B}^{n}\right)\right) / r^{\alpha}$ has a monotonicity property as in Theorems 1 and 2 or not.

4.3. The diameter version in the plane. We give a new proof of the following theorem, which implies the theorem of Landau and Toeplitz. We will use the Green function in place of the modulus metric. We denote by $g_{\Omega}$ the Green function for a domain $\Omega$.

Theorem 6 ([5]). Suppose that $f$ is holomorphic on $\mathbb{D}$. The function $\Phi_{D}(r):=$ $r^{-1} \operatorname{Diam}(f(r \mathbb{D}))$ is strictly increasing for $0<r<1$, except when $f$ is linear, in which case $\Phi_{D}$ is constant.

Proof. Let $0<r<s<1$. Let $\tilde{r}:=\operatorname{Diam}(f(r \mathbb{D})) / 2$ and $\tilde{s}:=\operatorname{Diam}(f(s \mathbb{D})) / 2$. Let $w_{r}, \tilde{w}_{r}$ be two points on $\overline{f(r \mathbb{D})}$ with $\operatorname{Diam}(\overline{f(r \mathbb{D})})=\left|w_{r}-\tilde{w}_{r}\right|$. We may assume that $w_{r}=-\tilde{r}$ and $\tilde{w}_{r}=\tilde{r}$. Let $z_{r}, \tilde{z}_{r}$ be points on the circle $\{|z|=r\}$ with $f\left(z_{r}\right)=-\tilde{r}$ and $f\left(\tilde{z}_{r}\right)=\tilde{r}$. By well-known properties of the Green function,

$$
g_{f(s \mathbb{D})}(-\tilde{r}, \tilde{r}) \geq g_{s \mathbb{D}}\left(z_{r}, \tilde{z}_{r}\right) \geq g_{s \mathbb{D}}(-r, r)=\log \left(\frac{1+r^{2} / s^{2}}{2 r / s}\right) .
$$

Let $\mathcal{S}_{1}, \mathcal{S}_{2}$ denote Steiner symmetrization with respect to the real and imaginary axes, respectively. Let $\Omega_{s}:=\mathcal{S}_{1} \circ \mathcal{S}_{2}(f(s \mathbb{D}))$. Since the Green function is increased by Steiner symmetrization (see [3]) and by domain expansion, we have

$$
g_{f(s \mathbb{D})}(-\tilde{r}, \tilde{r}) \leq g_{\Omega_{s}}(-\tilde{r}, \tilde{r}) \leq g_{\tilde{s} \mathbb{D}}(-\tilde{r}, \tilde{r})=\log \left(\frac{1+\tilde{r}^{2} / \tilde{s}^{2}}{2 \tilde{r} / \tilde{s}}\right) .
$$

By (4.3), (4.4), and the fact that the function $x \mapsto\left(1+x^{2}\right) /(2 x)$ is decreasing for $0<x<1$, we conclude that $\tilde{r} / \tilde{s} \leq r / s$. This implies that $\Phi_{D}$ is increasing.

Suppose that $\Phi_{D}(r)=\Phi_{D}(s)$ for some $0<r<s<1$. Then $\Phi_{D}(r)=\Phi_{D}(\rho)=$ $\Phi_{D}(s)$ for all $\rho$ with $r<\rho<s$. We must have equality in the Steiner symmetrization inequality for the Green function. Hence (see 4$]) f(s \mathbb{D}) \approx \tilde{s} \mathbb{D}$. Also, the first inequality in (4.3) becomes equality. It follows then from [11, Theorem 4.4.10] that $f$ maps the disk $s \mathbb{D}$ conformally onto $\tilde{s} \mathbb{D}$. So $f$ is linear.

\section{REFERENCES}

1. G.D. Anderson, M.K. Vamanamurthy, M. Vuorinen, Dimension-free quasiconformal distortion in n-space. Trans. Amer. Math. Soc. 297 (1986), no. 2, 687-706. MR854093 (87j:30039)

2. G.D. Anderson, M.K. Vamanamurthy, M. Vuorinen, Conformal Invariants, Inequalities, and Quasiconformal Maps. Wiley, 1997. MR1462077 (98h:30033)

3. A. Baernstein II, Integral means, univalent functions and circular symmetrization. Acta Math. 133 (1974), 139-169. MR0417406 (54:5456) 
4. D. Betsakos, Equality cases in the symmetrization inequalities for Brownian transition functions and Dirichlet heat kernels. Ann. Acad. Sci. Fenn. Math. 33 (2008), 413-427. MR 2431373 (2009e:60175)

5. R.B. Burckel, D.E. Marshall, D. Minda, P. Poggi-Corradini, T.J. Ransford, Area, capacity and diameter versions of Schwarz's lemma. Conform. Geom. Dyn. 12 (2008), 133-152. MR 2434356

6. V.N. Dubinin, Symmetrization in the geometric theory of functions of a complex variable. Russian Math. Surveys 49 (1994), 1-79. MR.1307130 (96b:30054)

7. W.K. Hayman, Multivalent Functions. Second edition. Cambridge University Press, 1994. MR.1310776 (96f:30003)

8. E. Landau, O. Toeplitz, Über die großte Schwankung einer analytischen Funktion in einem Kreise. Arch. der Math. und Physik (3) 11 (1907), 302-307.

9. O. Martio, V. Ryazanov, U. Srebro, E. Yakubov, Moduli in Modern Mapping Theory. Springer, 2009. MR2466579

10. O. Martio, S. Rickman, J. Väisälä, Definitions for quasiregular mappings. Ann. Acad. Sci. Fenn., Ser. A I 448 (1969). MR0259114 (41:3756)

11. T. Ransford, Potential Theory in the Complex Plane. Cambridge University Press, 1995. MR.1334766 (96e:31001)

12. R. Remmert, Classical Topics in Complex Function Theory. Springer-Verlag, 1998. MR:1483074 (98g:30002)

13. Yu. G. Reshetnyak, Space Mappings with Bounded Distortion, Translations of Mathematical Monographs, 73. American Mathematical Society, Providence, RI, 1989. MR994644 (90d:30067)

14. S. Rickman, Quasiregular Mappings. Springer-Verlag, 1993. MR1238941 (95g:30026)

15. J. Sarvas, Symmetrization of condensers in $n$-space. Ann. Acad. Sci. Fenn. Ser. A I, No. 522 (1972), 1-44. MR0348108 (50:606)

16. J. Väisälä, Lectures on n-Dimensional Quasiconformal Mappings. Lecture Notes in Math., 229, Springer-Verlag, 1971. MR0454009 (56:12260)

17. M. Vuorinen, Conformal Geometry and Quasiregular Mappings. Lecture Notes in Math., 1319, Springer-Verlag, 1988. MR950174 (89k:30021)

18. N. Zorii, Precise estimate of the 2-capacity of a condenser. Ukrainian Math. J. 42 (1990), 224-228. Translated from Ukrain. Mat. Zh. 42 (1990), 253-257. MR1053428 (91c:31003)

Department of Mathematics, Aristotle University of Thessaloniki, 54124 ThessaLONIKI, GREECE

E-mail address: betsakos@math.auth.gr 